Ornellas, A., Sánchez, J.A., Fraga, L. \& Domingo, L. (2015). Políticas y prácticas en la formación permanente del profesorado en TIC en Cataluña. Revista Electrónica Interuniversitaria de Formación del Profesorado, 18 (3), 83-96.

DOI: http://dx.doi.org/10.6018/reifop.18.3.190271

\title{
Políticas y prácticas en la formación permanente del profesorado en TIC en Cataluña
}

\author{
Adriana Ornellas ${ }^{(1)}$, Joan-Anton Sánchez ${ }^{(2)}$, Leticia Fraga Colman ${ }^{(2)}$, Laura Domingo ${ }^{(3)}$ \\ (1) Universitat Oberta de Catalunya \\ (2) Universitat de Barcelona \\ (3) Universitat de Vic
}

\section{Resumen}

En este artículo presentamos los resultados que emergen de una de las dimensiones investigadas en el proyecto "Políticas y prácticas en torno a las TIC en la enseñanza obligatoria: Implicaciones para la innovación y la mejora" (Ministerio de Ciencia e Innovación. SEJ2007-67562). Nos centramos aquí en uno de los factores determinantes del éxito o fracaso de las políticas destinadas a transformar las prácticas educativas a partir de la integración de las TIC: la formación inicial y permanente del profesorado.

\section{Palabras clave}

Formación del profesorado; TIC; políticas educativas; prácticas educativas.

\section{Policies and practices in teacher training in ICT in Catalonia}

\section{Abstract}

In this paper we present some of the results that emerge from the RDT project "Policy and Practice regarding ICT in Education: Implication for Educational Innovation and Improvement" (Ministerio de Educación y Ciencia SEJ2007-67562). We will focus on one of the factors that determine the success or failure of policies that intend to transform

\section{Contacto:}

Adriana Ornellas, aornellas@uoc.edu, Rambla del Poblenou 156. 08018 Barcelona. Tel: +34 93326 3540.

Artículo vinculado al proyecto "Políticas y prácticas en torno a las TIC en la enseñanza obligatoria: Implicaciones para la innovación y la mejora” (Ministerio de Ciencia e Innovación. SEJ2007-67562). 
educational practices from the integration of ICT: initial training and professional development of teachers.

\section{Key words}

Teacher training; ICT; educational policies; educational practices.

\section{La investigación}

En los últimos veinte años se han implementado, en el ámbito nacional e internacional, diversos planes y programas dirigidos a integrar las tecnologías de la información y la comunicación (TIC) en los sistemas educativos. Las razones que se apuntan para justificar el desarrollo de políticas educativas en el ámbito de las TIC pasan, entre otras, por la adecuación del sistema escolar a las características de la sociedad de la información; la preparación de los alumnos ante las nuevas formas culturales digitales; el incremento y mejora de la calidad de los procesos de enseñanza y aprendizaje o la innovación de los métodos y materiales didácticos.

No obstante, diversos estudios realizados en el contexto español, desde distintas comunidades autónomas, indican que pese a las diversas políticas institucionales dirigidas a la dotación y el uso de las TIC en los centros educativos, todavía no se ha alcanzado un nivel aceptable de generalización en el uso pedagógico de estas tecnologías por el profesorado de los diferentes niveles educativos (Cabero, 2000; De Pablos, Area, Valverde y Correa, 2009; Fernández y Cebreiro, 2002; Pérez Cota, Vilán y Machado da Costa, 2006; Sanabria, 2004). Muchos docentes utilizan las TIC como herramienta de trabajo para tareas de planificación de la enseñanza (búsqueda de información, preparación de actividades, presentación de la información a los estudiantes, etc.). Sin embargo, no han innovado su metodología y su práctica docente a partir de la adopción de las TIC como medio habitual de enseñanza. En Cataluña la situación no es distinta como se puede comprobar a partir de los estudios de Sigalés, Mominó y Meneses (2007), Law, Pelgrum y Plomp (2008) y Ornellas (2007).

Partiendo de estas premisas, el proyecto "Políticas y prácticas en torno a las TIC en la enseñanza obligatoria: Implicaciones para la innovación y la mejora" (Ministerio de Ciencia e Innovación. SEJ2007-67562)', se ha propuesto responder a los siguientes interrogantes:

- ¿Por qué a pesar de la existencia de programas específicos de introducción de las TIC en la enseñanza en la mayoría de los países su presencia en los centros suele ser insuficiente, anecdótica o no conlleva una innovación sustancial que signifique una mejora de los procesos y resultados del aprendizaje?

- ¿Qué tendría que cambiar en la política educativa, en los centros de enseñanza y en la formación docente para que profesorado y alumnado pudiera relacionarse de forma crítica, reflexiva y formativa con estas tecnologías y para que el sistema

\footnotetext{
1 Además de los autores de esta comunicación, los investigadores que forman parte de la investigación son: Cristina Alonso (coordinadora); Juana María Sancho; Alejandra Bosco; Silvina Casablancas; Karla Alonso; Óscar Moltó, Xavi Giró; Montse Guitert; Teresa Romeu; Jörg Müller y Paulo Padilla.
} 
educativo estuviese preparado para responder a las necesidades y desafíos educativos de la sociedad actual?

La finalidad principal del proyecto ha sido describir, analizar, interpretar y valorar la visión sobre la tecnología y la educación subyacente en las políticas y prácticas relacionadas con las TIC en la escuela, su grado de integración y sinergia con otras iniciativas políticas y su capacidad para impulsar la transformación, la innovación y la mejora de la educación obligatoria.

\section{Metodología}

La naturaleza compleja y social del problema investigado y los objetivos de la investigación han conllevado a la adopción de una dimensión ontológica y epistemológica de la investigación cercana al construccionismo (Guba y Lincoln, 1994). Esta perspectiva considera que toda la vida social es interpretativa y que todas las prácticas sociales son por definición 'significantes' y, por tanto, organizadas socialmente, conformadas de manera histórica y mediante realizaciones informadas políticamente. En este sentido, hemos adoptado la perspectiva crítica de análisis del discurso como marco metodológico que nos permitiría dar respuestas a los objetivos establecidos. El proyecto investiga, por tanto, el discurso que media en los textos y las acciones que reflejan las políticas institucionales planteadas en la Comunidad Autónoma de Cataluña y destinadas a facilitar la incorporación y uso de las TIC (Tecnologías de la Información y Comunicación) al sistema escolar en los últimos 20 años.

En la primera fase del proyecto se ha llevado a cabo un análisis de los discursos presentes en los documentos que reflejan las políticas en torno a la implementación de las TIC en los centros escolares de Cataluña. Para ello se han utilizado los métodos propuestos por Gee (1999). La estrategia clave en este proceso es focalizar los temas que emergen de un análisis de contenido inicial para luego ir desarrollando, de forma reflexiva la relación de estos temas con los discursos que fijan en torno a la tecnología, el aprendizaje, el profesorado, los estudiantes, la sociedad de la información y el cambio educativo. Se ha analizado la Legislación que ha regulado las políticas en torno a las TIC a lo largo de los últimos 25 años; las publicaciones institucionales relativas a estas políticas; los documentos que contienen las orientaciones para la aplicación de la normativa vigente sobre la organización y el funcionamiento de los centros educativos públicos de Cataluña; además de las webs institucionales. También se ha realizado un conjunto de entrevistas a dos grupos de personas clave del sistema educativo, quienes tienen responsabilidad en la elaboración y la implementación de las políticas (personal de la administración educativa) y quienes ven su trabajo afectado por ellas (profesorado y equipos directivos).

En la segunda fase se han realizado cuatro estudios de caso en cuatro centros públicos de Cataluña (dos de primaria y dos de secundaria), con el fin de analizar las dimensiones que caracterizan las experiencias reconocidas como innovadoras en el uso educativo de las TIC en los centros escolares. Se ha prestado especial atención a las visiones sobre el conocimiento y el aprendizaje, al papel del alumnado y el profesorado en los procesos de enseñanza y aprendizaje, a la función asignada a las TIC en el currículo, a la formación y la práctica del profesorado, entre otros. La realización de los estudios de caso ha comportado 
el análisis de documentos relevantes, entrevistas a informantes clave - que han sido grabadas y transcritas-, para explorar el fenómeno analizado de forma profunda, y la observación de la práctica y la dinámica del centro. Esta aproximación metodológica de carácter etnográfico (Denzin, 1997) es coherente con el planteamiento construccionista de la investigación, pues nos permite generar un conocimiento detallado sobre la dialéctica entre la actuación de los agentes educativos y las estructuras sociales de las que forman parte.

En este texto presentamos algunos de los resultados del proyecto relacionados con la dimensión de la formación del profesorado el ámbito de las TIC, que emergen del análisis cruzado de las evidencias obtenidas mediante los diferentes métodos e instrumentos de recogida, análisis e interpretación de la información.

\section{Resultados}

\subsection{La formación del profesorado en TIC en el marco del EEES}

La formación del profesorado, sea inicial o en ejercicio, se configura cada vez más como un elemento estratégico a la hora de impulsar el cambio educativo y dar respuesta a muchos de los nuevos retos y problemas de la educación contemporánea. El ingente desarrollo de las tecnologías digitales, y la facilidad en la generación, difusión y acceso a la información y el conocimiento, hacen imprescindible definir una serie de nuevas competencias para el ejercicio de la docencia. Éstas implican el uso de artefactos digitales, cuyo dominio comienza con su utilización, como instrumentos para tratar, almacenar y transmitir información; pero sobre todo como medios de enseñanza y aprendizaje, lo que implica también una visión crítica (Sancho, Ornellas, Sánchez, Alonso y Bosco, 2008).

Ante la necesidad de adquirir y desarrollar nuevos conocimientos y habilidades en un mundo digital, según las afirmaciones de los estudios realizados en el contexto español (Santoveña, 2012; Bernabé, 2012; Olivera y Agudo, 2012; Negre, Marín, Pérez, 2013), es fundamental el establecimiento de una estrecha relación entre los programas de formación inicial y permanente del profesorado por dos razones. La primera, para que los futuros educadores en su etapa de formación conozcan la realidad del sistema educativo. La segunda, para que los responsables de los programas de formación permanente prevean las necesidades formativas de los profesionales que se integran al sistema.

Sin embargo, tanto en Cataluña, como en otras Comunidades Autónomas de España, y pese a la reciente reforma del sistema universitario español, sigue perpetuándose el divorcio entre la formación inicial y la permanente del profesorado en competencias profesionales relacionadas con el uso pedagógico de las TIC.

Con el proceso de reforma general de los títulos y planes de estudio de la universidad española de la década de los noventa, los currículos de formación inicial de los educadores pasaron a contemplar una asignatura troncal de formación en el ámbito de las TIC. Hasta las reformas de los títulos universitarios para adaptarse a los requisitos del Espacio Europeo de Educación Superior (EEES), esta formación se concretaba en las titulaciones de formación de maestros en la asignatura troncal Nuevas Tecnologías Aplicadas a la Educación (NTAE), una asignatura de 4,5 créditos, común a todas las especialidades del título de maestro y que los estudiantes cursaban en el primer semestre del primer curso de los estudios. 
La ubicación de la asignatura en el primer semestre de los estudios, cuando los estudiantes todavía no han adquirido los conocimientos sobre didáctica necesarios para entender las implicaciones derivadas la introducción de las TIC en el aula, respondía a una concepción instrumentalista, centrada en la tecnología, latente en aquellos momentos y que pretendía introducir a los estudiantes en la cultura informática y capacitarlos como meros usuarios de la tecnología.

El reciente proceso de reforma de la enseñanza universitaria y de diseño e implantación de los nuevos grados impulsados por el EEES, ha dibujado un nuevo mapa en lo referente a la formación inicial del profesorado en el ámbito de las TIC en las nuevas titulaciones de formación de maestros (Olivera y Agudo, 2012). El tema de las competencias en relación a las TIC ha suscitado un importante debate, recogido de forma desigual por las diferentes universidades que tienen una cierta autonomía a la hora de decidir la configuración final de los planes de estudios.

La Red Universitaria de Tecnología Educativa $\left(\operatorname{RUTE}^{2}\right)$ y la Asociación para el desarrollo de la Tecnología Educativa y las Nuevas Tecnologías aplicadas a la Educación (EDUTEC ${ }^{3}$ ) han realizado una declaración conjunta en la que destacaban la importancia de garantizar una formación básica en TIC en todos los títulos de formación del profesorado (tanto de Infantil, Primaria como Secundaria). De acuerdo al documento:

"La formación del profesorado en competencias relacionadas con las tecnologías de la información y comunicación en contextos educativos es una necesidad formativa fundamental y relevante del profesorado del siglo XXI. Ante la complejidad de los problemas educativos que implican las TIC la formación del profesorado no debe tener que ver exclusivamente con el manejo de los diferentes desarrollos, sino que debe tender a propiciar un conocimiento que les permita la inserción de estas tecnologías en su práctica escolar." (RUTE, 2008)

En consecuencia, un modelo de formación integral de los futuros profesores en relación con las TIC debiera abarcar el desarrollo de las siguientes ámbitos de competencias: (a) Competencias instrumentales informáticas; (b) Competencias para el uso didáctico de la tecnología; (c) Competencias para la docencia virtual; (d) Competencias socioculturales; (e) Competencias comunicacionales a través de TIC.

La nueva ordenación de las enseñanzas universitarias que regulan la planificación de los títulos universitarios de grado de Maestro de Educación Infantil ${ }^{4}$ y de Educación Primaria ${ }^{5}$, así como el Máster de Profesor de Educación Secundaria ${ }^{6}$, establecen objetivos formativos destinados a capacitar a los futuros docentes en el uso educativo de las TIC.

En relación a las titulaciones de Maestro, se incluye como objetivo de la formación inicial de los maestros de la etapa de Educación Infantil: "conocer las implicaciones educativas de las tecnologías de la información y la comunicación y, en particular, de la televisión en la primera infancia" y de Maestro de Educación Primaria: "Conocer y aplicar en las aulas las tecnologías de la información y de la comunicación. Discernir selectivamente la información audiovisual

\footnotetext{
${ }^{2}$ Web de la red: http://www.rute.edu.es/

${ }^{3}$ Web de la asociación: http://www.edutec.es/

${ }^{4}$ Orden ECI/3854/2007. BOE de 29 de diciembre de 2007, núm. 312.

${ }^{5}$ Orden $\mathrm{ECl} / 3857 / 2007$. BOE de 29 de diciembre de 2007, núm. 312.

${ }^{6}$ Orden ECI/3858/2007. BOE de 29 de diciembre de 2007, núm. 312.
} 
que contribuya a los aprendizajes, a la formación cívica y a la riqueza cultural". Por otra parte en las directrices para la formación del profesorado de Educación Secundaria se establece como objetivo general el que estos futuros profesores sean capaces de "Buscar, obtener, procesar y comunicar información (oral, impresa, audiovisual, digital o multimedia), transformarla en conocimiento y aplicarla en los procesos de enseñanza y aprendizaje en las materias propias de la especialización cursada" (Ornellas y Sánchez, 2008).

Estos objetivos no se concretan en ningún modulo específico en TIC sino en competencias que se han de abordar en los diferentes módulos. Esta opción puede ayudar a los estudiantes a tener una visión más transversal de éstas, pero si no se concreta en los descriptores de las diferentes asignaturas que han de diseñar las diferentes universidades, puede comportar una menor competencia de los futuros graduados en este ámbito.

Por otro lado los objetivos y competencias que aparecen en la orden ministerial, parten de una visión básicamente instrumental de las TIC, desaprovechando el potencial que pueden tener éstas como facilitadores de procesos de innovación educativa. En este sentido se echan de menos vinculaciones de las TIC con el trabajo colaborativo y la construcción del conocimiento. Al tiempo que se desconoce la influencia del aprendizaje colaborativo a través de las TIC desde la gestión y dirección de los centros y cómo ello puede repercutir favorablemente en la formación del profesorado (González, Recamán y González, 2013). En otro orden sorprende la ausencia de competencias relacionadas con las TIC en los ámbitos disciplinares de la didáctica de la lengua, y que éstas se circunscriban únicamente al ámbito oral y escrito, cuando las necesidades formativas en nuestra sociedad hacen imprescindible la competencia en los nuevos alfabetismos (Sancho, 2006).

Si esto es así para los profesionales de la Educación Infantil y Primaria que cuentan con un sistema de formación inicial, cuando nos acercamos a la formación inicial del profesorado de Educación Secundaria, la formación pedagógica en el ámbito de las TIC sigue destacando por su ausencia en el proceso de substitución del antiguo Certificado de Aptitud Pedagógica (CAP) por el Máster de Formación del Profesorado de Secundaria.

En una entrevista colectiva realizada en el año 2008, en el marco del proyecto de investigación a los miembros de la Dirección General de Innovación (DGI) del Departamento de Educación de Catalunya, el subdirector general de Desarrollo y Formación Personal Docente (SGDP) resaltaba la problemática de la deficitaria formación inicial del profesorado en el ámbito de las TIC:

“... hay alumnos nativos digitales que han nacido con esta generación en la cual utilizan las tecnologías como usuarios y que al estudiar Magisterio no incorporan todos estos conocimientos [...] cuando llegan a su formación de Magisterio o del CAP no acaban de integrar esto y acaban siendo unos inmigrantes digitales como profesor [...] o sea, algo pasa, probablemente tiene mucho que ver con esto, de qué manera se gestiona el conocimiento, se gestiona el aprendizaje, los entornos en red, lo que es hacer pedagogía y el hacer didáctica, quiero decir como lo repiensan desde una perspectiva de las tecnologías del aprendizaje y el conocimiento..." [Subdirector general del SGDP]

La necesidad de una adecuada formación inicial del profesorado en competencias vinculadas a los nuevos medios y lenguajes de la sociedad digital ha sido puesta de manifiesto en diferentes estudios e informes internacionales. Referencias fundamentales 
son el informe EURYDICE (2002) que ofrece propuestas de mejora en la formación inicial del profesorado para responder a las nuevas demandas de la sociedad contemporánea o las directrices de la UNESCO sobre competencias en TIC para docentes (UNESCO, 2008). Pese a ello, la realidad de las prácticas en la formación inicial del profesorado en muchas instituciones universitarias sigue esquivando las recomendaciones de los expertos y de la propia legislación educativa.

\subsection{Un recorrido histórico por las políticas de formación permanente del profesorado en TIC en Cataluña}

La historia de la formación del profesorado en TIC en Cataluña está vinculada a la propia evolución de las políticas de integración de las tecnologías en el campo de la educación en esta comunidad autónoma. En este sentido, podemos distinguir tres grandes y marcadas etapas que coinciden con períodos de diez años.

La década de los 80 a los 90, es una primera fase en la que empiezan las experiencias piloto en los centros. En el año 1983 se inicia una serie de actividades en el campo de la informática en la enseñanza no universitaria, a modo de programas experimentales. Entre ellas se pueden citar la Experiencia Piloto de Informática Básica (EPIB) en el ámbito de la Formación Profesional y el Bachillerato y, en el ámbito de la enseñanza primaria, la experiencia de Enseñanza Asistida por Ordenador (EAO) dirigida al aprendizaje de las matemáticas y el Plan Experimental de Introducción al Lenguaje LOGO.

Todas estas experiencias suscitaron la necesidad de dotar de un carácter institucional las diversas iniciativas existentes en la aplicación educativa de las tecnologías informáticas. Como consecuencia, el Departamento de Educación del Gobierno de Cataluña creó en 1986 el Programa de Informática Educativa (PIE), que asumió la coordinación de las experiencias existentes e inició las actuaciones generalizadas especialmente en la Enseñanza Secundaria (PNTIC, 1991).

Con la creación del PIE tienen inicio también las acciones generalizadas de formación permanente del profesorado en el ámbito de las TIC impulsadas por la Administración educativa de Cataluña. Esta formación se ha llevado a cabo a lo largo de las últimas décadas bajo las directrices que han marcado los consecutivos "Planes de Formación Permanente del profesorado". La formación del profesorado en este período, es entendida como un proceso de cualificación técnica del profesorado necesario para facilitar la generalización del uso de las TIC en las escuelas. Desde esta perspectiva se presupone que con el conocimiento instrumental de las herramientas tecnológicas se producirá de manera automática un uso pedagógico de estos recursos por parte de los docentes. Con respecto a la perspectiva conceptual de formación permanente, predomina una visión técnica sobre la enseñanza y la función del profesorado centrada en modelos de formación de entrenamiento que pretenden formar a los docentes como usuarios eficaces de las TIC, visión que es presente en otras comunidades autónomas (Fernández, Rodríguez y Vidal, 2007). En este sentido, los cursos centrados en el conocimiento de los aspectos técnicos son la modalidad de formación más extendida (Ornellas, 2007).

La segunda década, de los 90 al año 2000 tiene como punto de inflexión la aprobación de la Ley Orgánica General del Sistema Educativo (LOGSE) que introduce como uno de sus 
principios básicos la educación permanente con el fin de favorecer la calidad de la enseñanza. También es un hito la aparición de Internet. Como afirmaba el Responsable del Servicio de Tecnologías para el Aprendizaje y el Conocimiento (STAC) del Departamento de Educación de Cataluña en entrevista realizada en el año 2008:

"entonces nos concentramos todos en al aprendizaje constructivista, explicar, vender la reforma, etc. y se abre una pequeña ventana en el 92, cuando se aprueban los decretos del currículo, para empezar ya una serie de aportaciones, para que en las distintas áreas del currículo se fueran introduciendo las tecnologías. [...] luego aquí en medio nace Internet, pero creo que la palabra que marca esta segunda oleada es la palabra de integración, de integración curricular, o se había que sembrar el currículo con elementos de tecnología. En la anterior década sobre todo nos concentrábamos en aprender o enseñar sobre tecnología [...] y y aquí (la segunda década) se trataba más bien de aprender de la tecnología[... ]." [Responsable del STAC]

El análisis de los objetivos y concreciones prácticas de la formación del profesorado en TIC en este período demuestra que sigue latente una forma de entender la integración curricular de las TIC como elemento de cambio e innovación educativa que se acerca a una perspectiva tecnológica sobre y para la innovación educativa (Ornellas, 2007). Desde esta perspectiva, los artefactos tecnológicos son el eje nuclear de las transformaciones y reformas educativas, casi un talismán con la propiedad de impulsar la renovación pedagógica en el conjunto del sistema educativo. Una perspectiva que conecta con una forma de entender las TIC como un instrumento en sí, que mejora automáticamente cualquier proceso en el que participen. Por otra parte, pese al énfasis "constructivista" de la reforma, la integración de las TIC en los currículos y la formación del profesorado sigue basándose en los usos reproductores de los medios, es decir "aprender de la tecnología".

Entre los años 2000 y 2010 , empieza una nueva etapa en la historia de las políticas educativas en TIC en Catalunya. A lo largo de esta década el Departamento de Educación pasa por tres procesos de reestructuración interna. La primera, sufrida en el año $2000^{7}$, concede un papel relevante a las TIC mediante la creación de la Subdirección General de Tecnologías de la Información (SGTI), adscrita a la Dirección General de Ordenación e Innovación Educativa, que pasa a englobar el antiguo PIE y el Programa de Medios Audiovisuales, que ahora dejan de ser "programas" y pasan a constituirse en el "Servicio de Informática Educativa y Académica" y "Servicio de Medios Audiovisuales".

En el año 2005, y coincidiendo con la aprobación de la LOE, el Departamento de Educación pasa por una nueva reestructuración y crea el Área TIC para aglutinar la globalidad de todos los sistemas de información, tanto de gestión, como de los empleados para la actividad docente. De esta forma se redefine el SGTI, que pierde su status de "Subdirección General" y se incorpora al Área TIC que pasa a ocuparse de la informática educativa en coordinación con la Dirección General de Ordenación e Innovación Educativa.

Pero es el decreto de reestructuración del Departamento aprobado a finales del año $2007^{8}$, el que supone un cambio radical en la concepción y el discurso de la administración educativa en torno a las TIC. Con la creación del STAC el discurso de la administración

\footnotetext{
7 Decreto 320/2000. DOGC de 10 de octubre de 2000, núm. 3242

${ }^{8}$ Decreto 269/2007. DOGC de 13 de diciembre de 2007, núm. 5026
} 
situaba en igualdad de condiciones los aspectos técnicos y pedagógicos asociados al discurso TIC. Un gran eje del cambio en la descripción de la nueva política de formación tiene que ver con el binomio TIC-TAC ${ }^{9}$, haciendo una clara apuesta por éstas últimas:

“... yo insistí mucho en separar todo lo que es equipamientos, lo que es el software, lo que es la educación, los ordenadores en los centros, lo que es en fin toda la parte esta más de máquina de hierro si quieres, lo que debe ser el uso didáctico, el uso metodológico, digamos de estas tecnologías para el aprendizaje y el conocimiento. (...) nos pondremos en estos aspectos más didácticos o más relacionados con la metodología, con la innovación, con la enseñanza y con el aprendizaje." [Director General de la DGI].

En la entrevista que realizamos a los nuevos responsables de la DGI del Departamento de Educación en el año 2008, se percibe una clara intencionalidad de marcar distancias con el discurso que durante más de veinte años ha prevalecido en el Departamento de Educación. En palabras del subdirector general del SGDP:

"Una de las cosas que es evidente es que en veinte años de TIC's en la educación, y además el informe PIC en Cataluña lo dice claramente, los alumnos y el profesorado son los usuarios más cualificados de las TIC, pero no en los ámbitos de la enseñanza y el aprendizaje, que es una cosa muy curiosa. Algo no funciona y algo se debe hacer para cambiar el modelo pedagógico del aula, porque está claro que con este modelo pedagógico que tenemos de la pizarra, los niños y las niñas mirándola, centrado en el profesor... las TIC aquí continúan siendo [...] una manera de escribir aquello que se hacía en papel haciéndolo a través del teclado, pero quiero decir que no es integrar, no se ha integrado esto se ha añadido, es un añadido." [Subdirector general del SGDP].

Centrándonos en la formación permanente del profesorado, los responsables de la nueva Administración presentan una nueva concepción de la formación, diferenciándola claramente de la llevada a cabo hasta el momento que califican de conductista, con materiales empaquetados, centralizada y napoleónica.

"[...] mientras la Subdirección General de Formación iba haciendo la formación presencial, el área TIC o la antigua Subdirección General de Tecnologías de la Información hacía su formación, llegó a un punto que [...] mientras nosotros teníamos un paradigma socio-constructivista, los otras estaban en el más puro conductismo [...] nosotros hemos visto como la formación no puede ser entendida de esta manera, la formación es un otra cosa, debemos partir de las necesidades del profesorado, debemos ver en qué punto se encuentran y ver como los ayudamos." [Director General de Innovación]

El plan de formación permanente del profesorado 2005-2010, marca un punto de inflexión en lo que se refiere al protagonismo dado a las TIC en los procesos de enseñanza y de aprendizaje, poniendo el énfasis en las posibilidades educativas que ofrecen las TIC contextualizadas en distintas formas de entender el currículum y en diferentes modelos pedagógicos. El documento que regula el plan contiene orientaciones para una formación del profesorado en TIC desde un modelo de desarrollo y mejora que contribuya a los

\footnotetext{
${ }^{9}$ Tecnologías de la información y la comunicación versus Tecnologías del aprendizaje y el conocimiento.
} 
procesos de cambio e innovación pedagógica a través de nuevas metodologías centradas en el alumnado, en la resolución de problemas, en el aprendizaje colaborativo y en la atención a la diversidad.

Tres son los ejes del cambio que introduce el plan. En primer lugar los planteamientos pedagógicos que sustentan los objetivos estratégicos de las acciones de formación, en segundo las nuevas prioridades formativas que se concretan en nuevos bloques en que se agrupan los cursos. $\mathrm{Y}$ finalmente los cambios por lo que respecta a las modalidades formativas. Los objetivos estratégicos del nuevo plan de formación, incorporan nuevas y esperanzadoras visiones de la educación, prácticamente inexistentes hasta el momento, vinculando currículo e innovación, situando al alumno como centro del aprendizaje, planteando utilizar las TIC de manera colaborativa, relacionando las ventajas del trabajo en red con la resolución de los problemas de la educación actual.

Teniendo en cuenta que, como afirmaba el director general de la DGI del Departamento de Educación, "la política se basa en este discurso, se fundamenta en estas nociones, en estos marcos conceptuales pero las prácticas tienen su ritmo de evolución que es otro", en el apartado siguiente analizaremos como estas políticas resuenan en las prácticas. Para ello, presentamos los resultados que emergen de los cuatro estudios de caso realizados en centros de educativos de Cataluña, respecto a la dimensión de la preparación y la formación del profesorado para la integración curricular de las TIC.

\subsection{Ecos en las prácticas educativas}

Como ya mencionamos anteriormente, en la segunda fase de la investigación se han realizado cuatro estudios de caso en centros públicos de Cataluña (dos de primaria y dos de secundaria), con el fin de elucidar el impacto en los centros escolares de las políticas de integración y uso de las TIC llevadas a cabo por la administración educativa en las últimas décadas. La realización de los estudios de caso ha comportado el análisis de documentos relevantes de los centros, entrevistas a informantes clave, entrevistas a estudiantes, así como la observación de la práctica y la dinámica del centro para explorar en profundidad el fenómeno analizado. Relataremos aquí algunos de los principales resultados encontrados relacionados con las prácticas, la formación y el desarrollo profesional del profesorado que son comunes a la realidad de estos centros.

La primera evidencia que emerge del análisis cruzado de los cuatro casos es que, pese a los diversos programas y políticas de integración de las TIC en los centros educativos y de las acciones de formación del profesorado llevadas a cabo por la administración educativa a lo largo de estas tres décadas, todavía no se ha alcanzado un nivel aceptable de generalización en el uso pedagógico de las TIC por el profesorado de los diferentes niveles educativos. Aunque muchos docentes utilizan las TIC como herramienta de trabajo para tareas de planificación de la enseñanza (búsqueda de información, preparación de apuntes o actividades, presentación de la información a los alumnos, etc), aún no han innovado su metodología y su práctica docente a partir de la adopción de las TIC como medio habitual de enseñanza (Sancho, 2008).

Gran parte de los docentes siguen considerando las TIC como nuevas tecnologías. En parte tienen razón, dado que estos medios siguen sin utilizarse de forma cotidiana en las prácticas de enseñanza pese a su presencia en los centros. No se han vuelto todavía “invisibles" como el libro de texto, la pizarra, el lápiz o el papel (Gros, 2000). 
En las entrevistas realizadas con los docentes de los centros de secundaria muchos manifestaban hacer un uso puntual de los recursos TIC del centro, sobre todo como forma de mostrar información al estudiante. Entre los principales obstáculos que dificultan un uso más extendido de las TIC en las clases suelen apuntar la falta de material adaptado a la asignatura que imparten, la falta de infraestructura adecuada en el instituto, la falta de formación para la utilización y la producción propia de materiales educativos.

En general, hemos encontrado en los centros un profesorado que todavía posee un dominio técnico muy básico de las TIC, pero también de las metodologías didácticas para trabajar en los nuevos entornos de enseñanza-aprendizaje.

"Yo, por ejemplo, la única formación que tengo respecto al tema, es la que hice en la universidad, que nos enseñaban a utilizar el PowerPoint para poder hacer presentaciones en clase. Básicamente yo he sido autodidacta, me ha interesado trabajar desde mi casa el tema de imágenes, me ha gustado trabajar mucho con el PowerPoint, trabajar con Word, pues bueno, voy descubriendo cosas" [Maestro centro Jl]

Desde los centros de primaria suelen existir muchas peticiones de ayuda, de asesoría, solicitudes de más y mejores cursos de formación en el uso de las TIC que posibiliten la generación de nuevas destrezas técnicas y didácticas. Formación, que, no sólo implique plantearse la utilización de los ordenadores en las clases, sino entender cómo un docente puede llegar a apropiarse e integrar esta amplia gama de recursos tecnológicos en el currículum escolar para facilitar el aprendizaje activo; o ayudarles a pensar dónde y cómo localizar los recursos que necesitan y gestionarlos. Paralelamente, también se critica la planificación de la formación por parte de la Administración educativa: "No siempre podemos acceder a los cursos que pedimos porque están llenos". [Maestro centro CG].

La no detección de las necesidades de formación y la inadecuación de las actividades de formación promovidas por el Departamento de Educación a las necesidades de los centros lleva a los propios centros muchas veces a compensar esta carencia a través de la organización de cursos internos. Algunos equipos directivos apuestan por una formación basada en el asesoramiento y la formación en el propio centro de trabajo, relacionada con el desarrollo de proyectos, como forma de fomentar la participación activa del profesorado en su propia formación y la aplicabilidad de lo aprendido en su práctica docente.

La meritocracia en la que se basa el sistema de promoción del profesorado incentiva, por otro lado, la formación como obtención de un título:

"Las políticas oficiales no han dado apoyo a estas cosas, como ejemplo podemos citar los cursos de diseño de páginas web, te dan el título, y te vas a casa, y con eso tienes tantos puntos [...]. La gente aprende cosas rarísimas que no tienen nada que ver con la experiencia didáctica [...] y como la gente necesita los puntos para los sexenios [...] pero sin perspectiva de aplicarlo, ni control de que se aplica [...]. Debería haber algún mecanismo para demostrar lo que has aprendido y que lo aplicas de alguna manera [...] Teóricamente el profesorado del país está formado, pero... este año se han reciclado no sé cuántos millones de profesores en nuevas tecnologías, pero..." [Director del Instituto SE] 


\section{Conclusiones}

Los resultados de la investigación demuestran que el discurso y la voluntad política de transformación de las prácticas educativas vinculadas a la introducción de las TIC en los procesos de enseñanza y aprendizaje resuenan todavía de manera muy tímida en la realidad de los centros educativos.

La desconexión entre los sistemas de formación inicial y permanente del profesorado en el ámbito de las TIC en Cataluña, así como la perspectiva tecnicista e instrumental que ha dominado esta formación contribuyen, en parte, a explicar la dificultad para alcanzar el primer objetivo del Programa de Informática Educativa, estipulado hace más de veinte años, y que consistía en "contribuir a la mejora del proceso de aprendizaje y favorecer el desarrollo de la capacidad de plantear y resolver problemas, la intuición y la creatividad".

El último plan de formación permanente marca un punto de inflexión en lo que se refiere al protagonismo dado a las TIC en los procesos de enseñanza y de aprendizaje, poniendo el énfasis en las posibilidades educativas que ofrecen estas tecnologías contextualizadas en distintas formas de entender el currículum y en diferentes modelos pedagógicos. Aunque no podemos predecir el futuro con precisión, consideramos que las nuevas directrices abren un nuevo camino esperanzador para que en la próxima década se pueda abordar la formación de los docentes en el ámbito de las TIC desde un complejo conjunto de factores sociales, culturales, pedagógicos, éticos, curriculares y organizativos que contribuyan a la emancipación del profesorado y a la transformación de las prácticas educativas.

\section{Referencias}

Bernabé, M. (2012). Análisis de las necesidades de la formación docente para contextos pluriculturales. Revista Interuniversitària de Formación del Profesorado, 15(4), 79-88. Recuperado desde http://www.redalyc.org/articulo.oa?id=217026243002

Cabero, J. (2000). Usos de los medios audiovisuales, informáticos y NNTT en los centros andaluces. Sevilla: Kronos.

De Pablos, J., Area, M., Valverde, J., y Correa, J.M. (2009). Autonomic educational policies and the integration of ICT in school centers. En A. Méndez-Vilas, A. Solano Martín, J.A. Mesa González y J. Mesa González (Eds.), Research, Reflections and Innovations in Integrating ICT in Education (p. 1275-1279). Badajoz: Formatex.

Denzin, N. (1997). Etnografía de la Interpretación: Prácticas Etnográficas para el siglo 21. Newbury Park, CA: Sage.

Eurydice (2002). Formación inicial y transición a la vida laboral. Madrid: CIDE.

Fernández, M.D. Rodríguez. J., y Vidal, M.P. (2007). TIC y desarrollo profesional del profesorado. El caso de un centro de primaria. Revista Interuniversitaria de Formación del Profesorado, 21, 85-110.

Fernández, C., y Cebreiro, B. (2002). La integración de los medios y nuevas tecnologías en los centros y prácticas docentes. Pixel-Bit. Revista de Medios y Educación, 20, 33-42.

Gee, J.P. (1999). An introduction to discourse analysis: theory and method. New York. Routledge. 
González, L.M., Recamán, A., y González, C. (2013). La dimensión colaborativa con TIC en la dirección de centros. Revista Electrónica Interuniversitaria de Formación del Profesorado, 16(1), 147-162. doi: 10.6018/reifop.16.1.179501

Gros, B. (2000). El ordenador invisible. Barcelona: Gedisa.

Guba, E.G. y Lincoln, Y. S. (1994). Competing paradigms in qualitative research. En Denzin, N. K. y Lincoln, Y. S. (eds.): Handbook of Qualitative Research. Sage Punblications. California, 105-117.

Law, N., Pelgrum, W.J., y Plomp, T. (2008). Pedagogy and ICT use in schools around the world: findings from the IEA SITES 2006 study. Hong Kong: CERC-Springer.

Negre, F., Marín, V.I., y Pérez, A. (2013). Estrategias para la adquisición de la competencia informacional en la formación inicial de profesorado de primaria. Revista Electrónica Interuniversitaria de Formación del Profesorado, 16 (2), 1-12. doi: 10.6018/reifop.16.2.180751

Oliveras, M.L., y Agudo, N. (2012). Evaluación de un modelo de formación inicial de profesores para su adaptación al EEES. Incidencia del Portafolio. Revista Electrónica Interuniversitaria de Formación del Profesorado, 15(4), 89-99. Recuperado desde http://revistas.um.es/reifop/article/view/174841

Ornellas, A. (2007). La formación permanente del profesorado de secundaria en tecnologías de la información y la comunicación: el caso de Cataluña (Tesis doctoral no publicada). Universidad de Barcelona.

Ornellas, A., y Sánchez, J.A. (2008). La formación del profesorado en Tecnologías de la Información y la Comunicación en Cataluña. Diálogos. Educación y formación de personas adultas, 55, 49-62.

Pérez, M., Vilán L., y Machado, J.P. (2006). Integración de las TIC en el sistema educativo de Galicia: respuesta de los docentes. RELATEC: Revista Latinoamericana de Tecnología Educativa, 5(2), 177-189.

PNTIC (1991). Las nuevas tecnologías de la información en la educación. Madrid: Ministerio de Educación y Ciencia.

RUTE (2008). La formación para el desarrollo de las competencias de los futuros profesores en el uso pedagógico de las TIC. Recuperado desde http://www.juntadeandalucia.es/averroes/ escuelatic20/msec/didactica/Declaracion RUTE2008.pdf.

Sanabria, A. (2004). La formación permanente del profesorado para la integración de las tecnologías de la información y la comunicación en la Comunidad Autónoma de Canarias (Tesis doctoral no publicada). Universidad de la Laguna, Tenerife.

Sancho, J.M. (2006). Formar lectores y autores en un mundo visual. Cuadernos de Pedagogía, 363, 52-57.

Sancho, J. M. (2008). De TIC a TAC, el difícil tránsito de una vocal. Investigación en la escuela, 64, 19-29. 
Sancho, J.M., Ornellas, A., Sánchez, J.A., Alonso, C. y Bosco, A. (2008). La formación del profesorado en el uso educativo de las TIC: una aproximación desde la política educativa. Praxis Educativa, 12, 22-28.

Santoveña, S. (2012). La formación permanente del profesorado en entornos virtuales de aprendizaje. Revista Electrónica Interuniversitaria de Formación del Profesorado, 15(4), 6977. Recuperado desde http://dialnet.unirioja.es/servlet/articulo?codigo=4614623

Sigalés, C.; Mominó, J. M. y Meneses, J. (2007). L'escola a la Societat Xarxa: Internet a l'educació Primària i Secundària. Informe final de recerca. Universitat Oberta de Catalunya. Recuperado desde http://www.uoc.edu/in3/pic/cat/escola_xarxa/informe.html.

UNESCO (2008). ICT competency standards for teachers. Recuperado desde http://cst.unesco-ci.org/sites/projects/cst/default.aspx. 


\section{Autores:}

Adriana Ornellas

Doctora en Pedagogía por la Universidad de Barcelona. Profesora de los estudios de Informática Multimedia y Telecomunicación de la UOC. Profesora asociada del Departamento de Didáctica y Organización Educativa de la Universitat de Barcelona. Miembro del grupo de investigación consolidado Esbrina (Subjectivitats i Entorns Educatius Contemporanis) y del grupo de innovación docente Indaga't de la Universidad de Barcelona. Investigadora vinculada al e-Learn Center de la UOC. Líneas de investigación: Entornos y tecnologías emergentes para el aprendizaje en la sociedad contemporánea; formación inicial y desarrollo profesional docente en TIC; múltiples alfabetismos y sistemas de inclusión en la sociedad de la información; enseñanza y aprendizaje colaborativos en entornos virtuales.

\section{Joan-Anton Sánchez}

Doctor en Pedagogía. Profesor del departamento de Didáctica y Organización Educativa de la Universidad de Barcelona. Miembro del grupo de investigación consolidado Esbrina, subjetividades y entornos educativos contemporáneos. Miembro de Indaga't, grupo de innovación docente consolidado para favorecer la indagación. Líneas de investigación: Innovación docente en la universidad, entornos virtuales de aprendizaje, tecnologías digitales en la educación.

\section{Leticia Fraga Colman}

Profesora Asociada del departamento de Didáctica y Organización Educativa de la Universidad de Barcelona. Integrante del grupo de investigación consolidado Esbrina, subjetividades y entornos educativos contemporáneos. Líneas de investigación: subjetividades emergentes y múltiples alfabetizaciones; jóvenes y aprendizaje; narrativas visuales y convergencia digital.

\section{Laura Domingo}

Maestra, psicopedagoga, Máster europeo en “Recerca en Didàctica, Formació i Avaluació Educativa" por la Universitat de Barcelona (UB) y Doctora en Pedagogía por la Universidad de Vic (Uvic). Profesora e investigadora contratada per la UVic. Miembro del Grupo de Investigación Consolidado GREUV de la Uvic (2014 SGR 1291). Líneas de investigación: educación inclusiva, escuela rural, didáctica multigrado, TIC e identidad docente. 